\title{
UNIFORM HYPERBOLICITY ALONG PERIODIC ORBITS
}

\begin{abstract}
ABBAS FAKHARI
(Communicated by Bryna Kra)

ABSTRACT. We introduce the notion of uniform hyperbolicity along periodic orbits (UHPO) for homoclinic classes and provide equivalent conditions under which the UHPO property on a $C^{1}$-generic homoclinic class implies hyperbolicity. It is shown that for a $C^{1}$-generic locally maximal homoclinic class the UHPO property is equivalent to the non-existence of zero Lyapunov exponents. Using the notion of UHPO, we also give new proofs for some recent $C^{1}$-dichotomy theorems.
\end{abstract}

\section{INTRODUCTION}

The concept of hyperbolicity has played a central role in the theory of dynamical systems during the last 50 years or more. Soon after the introduction of uniform hyperbolicity in the 60 's, it was observed that in many natural and important examples one needs to have a more flexible theory by defining weak forms of hyperbolicity. One of the most important weak forms is non-uniform hyperbolicity, introduced in the early 70's. Roughly speaking, non-uniform hyperbolicity is characterized by the existence of some regions with a non-uniform rate of hyperbolicity. Conceptually, a basic question in this respect is

\section{Under what conditions may non-uniform hyperbolicity be converted into uniform hyperbolicity?}

There are several plausible ways to answer this question, while some basic ideas go back to pioneering works. Among the examples of the recent works, one can refer to [4, 5, 10, 11, 13, 18, for the non-existence of zero Lyapunov exponents, 9] for uniform hyperbolicity along periodic orbits with one hyperbolic direction and 14 for uniform hyperbolicity along periodic orbits of a shadowable invariant set.

As is known, the hyperbolicity of an invariant compact set implies the hyperbolicity of the periodic points inside. But what can be said about the converse? How can one obtain the uniform hyperbolicity of an invariant compact set knowing the hyperbolicity of the periodic orbits in the set? Although persistent hyperbolicity of the periodic orbits in the ambient manifold implies the uniform hyperbolicity of a non-wandering set $(19,21)$, the answer is known to be negative in general. For instance, periodic orbits of a Kupka-Smale diffeomorphism are all hyperbolic, but a Kupka-Smale diffeomorphism isn't uniformly hyperbolic in general. A very useful tool in this respect has been introduced by Bonatti et al. in [9] through the notion

Received by the editors June 2, 2011 and, in revised form, September 27, 2011 and November $12,2011$.

2010 Mathematics Subject Classification. Primary 37B20, 37C29, 37C50.

Key words and phrases. Hyperbolicity along periodic orbits, homoclinic class, dominated splitting, uniform hyperbolicity. 
of "uniform hyperbolicity along periodic orbits". There, the authors have shown that if a homoclinic class $H(p, f)$ admits a dominated splitting with a contractive direction, then the uniform hyperbolicity along the periodic orbits homoclinically related to $p$ implies the hyperbolicity of $H(p, f)$. In fact, this is the essential motivation for our main definition. In this paper, we impose the same condition on a $C^{1}$-generic homoclinic class. Doing so, the uniform hyperbolicity of the homoclinic class is derived via some equivalent conditions. The novelty here is that we not only show the existence of a dominated splitting for a generic homoclinic class satisfying the condition mentioned above, but we also omit the assumption that one of the directions is contracting. We also illustrate the equivalence of the imposed condition and the non-existence of zero Lyapunov exponents for $C^{1}$-generic locally maximal homoclinic classes.

Statement of results. We start by introducing the most relevant concepts and definitions. Suppose that $M$ is a closed manifold equipped with a Riemannian metric $d$ and $f: M \rightarrow M$ is a diffeomorphism. For any two points $x, y \in M$, a finite sequence $\left\{x_{0}=x, x_{1}, \ldots, x_{n-1}=y\right\}$ is called a $\delta$-pseudo orbit from $x$ to $y$ if $d\left(f\left(x_{i}\right), x_{i+1}\right)<\delta$, for any $0 \leq i<n$. If $x=y$, then the pseudo orbit is called a periodic pseudo orbit of length $n$. We define the relation $\leadsto$ as follows: $x \leadsto y$ if for any $\delta>0$, there exists a $\delta$-pseudo orbit from $x$ to $y$ and from $y$ to $x$. A point $x$ is chain recurrent if $x \leftrightarrow x$; denote by $\mathcal{C} \mathcal{R}(f)$ the set of all chain recurrent points of $f$. The relation $m$ induces on $\mathcal{C} \mathcal{R}(f)$ an equivalence relation whose classes are called chain components of $f$.

A pseudo orbit $\left\{x_{i}\right\}_{i=0}^{n}$ is $\epsilon$-shadowed by a point $x$ if $d\left(f^{i}(x), x_{i}\right)<\epsilon$, for any $0 \leq i \leq n$. An invariant compact set $\Lambda$ has the shadowing property if for any $\epsilon>0$ there is a $\delta>0$ such that any $\delta$-pseudo orbit in $\Lambda$ can be $\epsilon$-shadowed by some point in $M$. The set $\Lambda$ has the (inner) periodic shadowing if any periodic pseudo orbit of $f$ of length $n$ in $\Lambda$ can be shadowed by some periodic point of $f$ in $\Lambda$ of period $n$.

A closed $f$-invariant set $\Lambda \subset M$ is called hyperbolic if the tangent bundle $T_{\Lambda} M$ has a $D f$-invariant splitting $E^{s} \oplus E^{u}$ such that for some constants $C>0$ and $0<\lambda<1$,

$$
\left\|\left.D f^{n}\right|_{E^{s}(x)}\right\| \leq C \lambda^{n} \text { and }\left\|\left.D f^{-n}\right|_{E^{u}\left(f^{n}(x)\right)}\right\| \leq C \lambda^{n}
$$

for any $x \in \Lambda$ and any $n \geq 0$. Moreover, we say that $\Lambda$ admits a dominated splitting if the tangent bundle $T_{\Lambda} M$ has a $D f$-invariant splitting $E \oplus F$ such that for some constants $C>0$ and $0<\lambda<1$,

$$
\left\|\left.D f^{n}\right|_{E(x)}\right\| \cdot\left\|\left.D f^{-n}\right|_{F\left(f^{n}(x)\right)}\right\| \leq C \lambda^{n}
$$

for any $x \in \Lambda$ and any $n \geq 0$. It is well known that the two directions $E$ and $F$ are continuous (see appendix B in [8]). We recall that a direction $E \subset T M$ is continuous if it is possible to guarantee that the two unit balls of $E(x)$ and $E(y)$ be close to each other in Hausdorff metric by requiring that $x$ and $y$ are sufficiently close to each other.

For a hyperbolic periodic point $p$ of a diffeomorphism $f$ we denote by $\pi(p)$ its period. The two sets

$$
\begin{gathered}
W^{s}(p)=\left\{x \in M: f^{\pi(p) n}(x) \rightarrow p \text { as } n \rightarrow \infty\right\} \text { and } \\
W^{u}(p)=\left\{x \in M: f^{-\pi(p) n}(x) \rightarrow p \text { as } n \rightarrow \infty\right\}
\end{gathered}
$$

are injectively immersed submanifolds of $M$. A point $x \in W^{s}(p) \cap W^{u}(p)$ is a homoclinic point of $f$ associated to $p$, and it is a transversal homoclinic point if 
the intersection above is transversal at $x$. The closure of the set of all transversal homoclinic points of $f$ associated to the orbit of $p$ is called the transversal homoclinic class of $f$ associated to $p$ and is denoted by $H(p, f)$. Two saddles $p$ and $q$ are homoclinically related, and we write $q \sim p$ if

$$
W^{s}(\mathcal{O}(p)) \pitchfork W^{u}(\mathcal{O}(q)) \neq \phi \quad \text { and } W^{u}(\mathcal{O}(p)) \pitchfork W^{s}(\mathcal{O}(q)) \neq \phi .
$$

By Smale's Transverse Homoclinic Point Theorem, $H(p, f)$ coincides with the closure of the set of all hyperbolic periodic points which are homoclinically related to $p$.

Definition 1.1. For a saddle $p$ of a diffeomorphism $f$, we say that $f$ is uniformly hyperbolic along the periodic orbits homoclinically related to $p$ (or briefly, $f$ has UHPO property on $H(p, f))$ if there exist a constant $0<\lambda<1$ and a natural number $k$ such that for any periodic point $q$ which is homoclinically related to $p$,

$$
\prod_{i=0}^{\pi(q)-1}\left\|\left.D f^{k}\right|_{E^{s}\left(f^{i}(q)\right)}\right\|<\lambda^{\pi(q)} \text { and } \prod_{i=0}^{\pi(q)-1}\left\|\left.D f^{-k}\right|_{E^{u}\left(f^{i}(q)\right)}\right\|<\lambda^{\pi(q)} .
$$

In other words, the UHPO property indicates the absence of the weak hyperbolicity along periodic orbits homoclinically related to $p$.

We recall that a compact invariant subset $\Lambda$ is locally maximal if there is a neighborhood $U$ of $\Lambda$ such that $\Lambda=\bigcap_{n \in \mathbb{Z}} f^{n}(U)$. A subset $\mathcal{R}$ of a complete space $X$ is residual if it contains a countable intersection of open and dense subsets of $X$. Now, we can formulate the main goal of the present paper as follows.

Main Theorem. There is a residual subset $\mathcal{R}$ of $\operatorname{Diff}^{1}(M)$ such that for any $f \in \mathcal{R}$ and any saddle $p$ of $f$, if $f$ has UHPO property on $H(p, f)$, then the following three conditions are equivalent:

(a) $H(p, f)$ is hyperbolic,

(b) $H(p, f)$ has the periodic shadowing property,

(c) $H(p, f)$ is locally maximal.

Finally, we also see that the notion of UHPO can be adapted almost straightforwardly to give new proofs for some recent $C^{1}$-generic dichotomy theorems for locally maximal chain components (see section 3 ).

\section{Proof of the Main Theorem}

By a known result, there is a residual subset of $\operatorname{Diff}^{1}(M)$ for which the homoclinic class of a hyperbolic saddle $p$ coincides with the chain component containing $p$. In particular, hyperbolic homoclinic classes of such diffeomorphisms are locally maximal $([6)$. Hence, the two parts $(\mathrm{a}) \Rightarrow(\mathrm{b})$ and $(\mathrm{a}) \Rightarrow(\mathrm{c})$ of the Main Theorem hold $C^{1}$-generically. In the remainder of the paper, we dedicate our attention to concluding uniform hyperbolicity from the periodic shadowing and local maximality conditions. Recall that the index of a hyperbolic periodic point is the dimension of its stable direction.

Before continuing, let us explain how the UHPO property on a $C^{1}$-generic homoclinic class can be used to obtain a dominated splitting $E \oplus F$ with $\operatorname{dim} E=$ $\operatorname{index}(p)$. This fact is essential in the rest of the paper.

Given $\eta>0$, we say that a saddle $p$ of period $\pi(p)$ of a diffeomorphism $f$ is $\eta$-weakly hyperbolic if $D f^{\pi(p)}$ has an eigenvalue $\lambda$ with

$$
(1-\eta)^{\pi(p)}<|\lambda|<(1+\eta)^{\pi(p)} \text {. }
$$


If $p$ is a hyperbolic periodic point of $f$, then, for every $g C^{1}$-close to $f$, there is a hyperbolic periodic point $p_{g}$ of $g$ close to $p$, called the continuation of $p$ for $g$. The following remarkable result is obtained in [30].

Proposition 2.1. There is a residual subset $\mathcal{R}_{1}$ of $\operatorname{Diff}^{1}(M)$ such that for any $f \in \mathcal{R}_{1}$ and any $\eta>0$, if any neighborhood of $f$ contains a diffeomorphism $g$ having an $\eta$-weak hyperbolic saddle in $H\left(p_{g}, g\right)$ and of the same index as that of $p$, then $f$ has also a hyperbolic saddle in $H(p, f)$ which is $2 \eta$-weak hyperbolic and has the same index as that of $p$.

Now, consider a diffeomorphism $f \in \mathcal{R}_{1}$ having the UHPO property on a homoclinic class $H(p, f)$. Using Proposition 2.1 and repeating the process used in 30, one can extend the UHPO property to a neighborhood of $f$ in the $C^{1}$-topology. More precisely, there is a $C^{1}$-neighborhood $\mathcal{U}$ of $f$ such that any $g \in \mathcal{U}$ has the UHPO property on the homoclinic class $H\left(p_{g}, g\right)$, for some constant $\lambda$ independent to the choice of $g$. In this process, one can also obtain inequalities of the form

$$
\prod_{i=0}^{[\pi(q) / k]-1}\left\|\left.D f^{k}\right|_{E^{s}\left(f^{i k}(q)\right)}\right\|<\lambda^{[\pi(q) / k]} \text { and } \prod_{i=0}^{[\pi(q) / k]-1}\left\|\left.D f^{-k}\right|_{E^{u}\left(f^{i k}(q)\right)}\right\|<\lambda^{[\pi(q) / k]},
$$

for any periodic point $q$ which is homoclinically related to $p$. In particular, up to replacing $f^{k}$ by $f$ in the equations above, we can (and we do) assume $k=1$ in Definition 1.1. The existence of the desired dominated splitting involves some adaptations of the constructions in [21, 29, 30]. Let us summarize the discussion above in the following proposition.

Proposition 2.2. For any $f \in \mathcal{R}_{1}$ and any saddle $p$ of $f$, the UHPO property on $H(p, f)$ implies the existence of a dominated splitting $E \oplus F$ for $H(p, f)$ with $\operatorname{dim} E=\operatorname{index}(p)$.

\section{Remark 2.3. Dissipative and Two Dimensional Cases.}

\section{- Dissipative Case}

A diffeomorphism $f$ is dissipative on an invariant compact set $\Lambda$ if there is a $0<b<1$ such that for any $x \in \Lambda$, $\operatorname{det}(D f(x)) \leq b$. If $f \in \mathcal{R}_{1}$ is dissipative and has the UHPO property on a homoclinic class $H(p, f)$ with $\operatorname{index}(p)=1$, then the homoclinic class is hyperbolic. In fact, if $E \oplus F$ is the dominated splitting given by Proposition 2.2, then the direction $E$ will be immediately contracting ([26]). The expansion of the second direction can be obtained from [9].

\section{- Two Dimensional Case}

(1) Due to dimension deficiency, for a surface diffeomorphism $f \in \mathcal{R}_{1}$, the UHPO property on a locally maximal homoclinic class is characterized as hyperbolicity. This follows immediately from Mañé's method ([21]).

(2) Recently, Pujals and Sambarino [25] have proved a dichotomy for the dynamics of a non-wandering invariant set of a $C^{2}$ surface diffeomorphism having dominated splitting. More precisely, they have proved that if $f \in \operatorname{Diff}^{2}\left(M^{2}\right)$ is a Kupka-Smale diffeomorphism and $\Lambda$ is a compact invariant set contained in the limit set and exhibiting a dominated splitting, then it can be decomposed into a hyperbolic set and a finite number of periodic closed curves normally hyperbolic with dynamic conjugated to an irrational rotation. Assuming the shadowing property 
of $f$ on the set $\Lambda$, one can see easily that the normally hyperbolic periodic closed curves do not exist ([27]). Consequently, for a $C^{2}$-surface diffeomorphism $f \in \mathcal{R}_{1}$ having the UHPO property on a homoclinic class, the shadowing (not necessarily periodic shadowing) property is equivalent to hyperbolicity.

2.1. From periodic shadowing to hyperbolicity, $(\mathbf{b}) \Rightarrow(\mathbf{a})$. We begin this section by a simple remark on the behavior of a homoclinic class having the shadowing property. We give the proof for completeness.

Lemma 2.4. If $f$ has the shadowing property on a homoclinic class $H(p, f)$, then for any hyperbolic periodic point $q \in H(p, f)$,

$$
W^{s}(p) \cap W^{u}(q) \neq \emptyset \text { and } W^{u}(p) \cap W^{s}(q) \neq \emptyset .
$$

Proof. For given $\epsilon>0$, let $\delta$ be an $\epsilon$ modulus of the shadowing property. Choose a $\delta$-pseudo orbit $\xi$ in $H(p, f)$ from $p$ to $q$. Suppose that the $\delta$-pseudo orbit $\left\{\ldots, f^{-1}(p), \xi, q, f(q), \ldots\right\}$ is $\epsilon$-shadowed by some point, say $x$. By the Stable Manifold Theorem, $x \in W^{s}(p) \cap W^{u}(q)$. A same argument shows that $W^{u}(p) \cap W^{s}(q) \neq$ $\emptyset$.

Remark 2.5. As a consequence of Lemma 2.4, if a Kupka-Smale diffeomorphism $f$ has the shadowing property on a homoclinic class $H(p, f)$, then any saddle $q \in$ $H(p, f)$ is homoclinically related to $p$.

Having proven this preliminary lemma, we turn to the proof of $(b) \Rightarrow(a)$. Consider a Kupka-Smale diffeomorphism $f \in \mathcal{R}_{1}$ having the UHPO and periodic shadowing properties on a homoclinic class $H(p, f)$. Since $f \in \mathcal{R}_{1}$, the homoclinic class $H(p, f)$ admits a dominated splitting $E \oplus F$ with $\operatorname{dim} E=\operatorname{index}(p)$. Let us display a brief outline of the proof of contraction of the direction $E$; the expansion of the direction $F$ can be obtained similarly.

Choose $\lambda_{0}<\lambda<1$ and $0<\gamma<1$ with $\lambda_{0}(1+\gamma)<\lambda$. There is a positive $\delta$ such that $d(x, y)<\delta$ implies that

$$
1-\gamma<\frac{\left\|\left.D f\right|_{E(x)}\right\|}{\left\|\left.D f\right|_{E(y)}\right\|}<1+\gamma .
$$

It suffices to prove that for any $x \in H(p, f), \liminf _{n \rightarrow \infty} \log \left\|D f_{E\left(f^{n}(x)\right)}^{n}\right\|<0$ ([21]). By contradiction, assume that there is a point $z \in H(p, f)$ such that

$$
\liminf _{n \rightarrow \infty} \log \left\|\left.D f\right|_{E\left(f^{n}(z)\right)}\right\| \geq 0 .
$$

Hence, there is a sequence $k_{n}$ of natural numbers such that

$$
\frac{1}{k_{n}} \log \left\|\left.D f^{k_{n}}\right|_{E(z)}\right\|>\log \lambda .
$$

By Rieze's Representation and Birkhoff's Ergodic Theorems, there is an $f$-invariant probability measure $\mu$ such that (Theorem B in [21])

$$
\int_{H(p, f) \cap \mathcal{R}(f)} \lim _{n \rightarrow \infty} \frac{1}{n} \sum_{i=0}^{n-1} \log \left\|\left.D f\right|_{E\left(f^{i}(x)\right)}\right\| d \mu>\log \lambda .
$$

Thus, there is a recurrent point $y \in H(p, f)$ and a positive integer $n_{0}$ such that

$$
\frac{1}{n} \sum_{i=0}^{n-1} \log \left\|\left.D f\right|_{E\left(f^{i}(y)\right)}\right\|>\log \lambda,
$$


for any $n \geq n_{0}$. Choose $n \geq n_{0}$ such that $d\left(f^{n}(y), y\right)<\eta$, where $\eta$ is a $\delta$ modulus of the periodic shadowing property. Let $q \in H(p, f)$ be a periodic point which $\delta$-shadows the periodic pseudo orbit $\left\{y, \ldots, f^{n}(y)\right\}$. We should notice that by Remark 2.5, $q \sim p$. On the other hand,

$$
\lambda^{n}<\prod_{i=0}^{n-1}\left\|\left.D f\right|_{E\left(f^{i}(y)\right)}\right\| \leq(1+\gamma)^{n} \prod_{i=0}^{n-1}\left\|\left.D f\right|_{E^{s}\left(f^{i}(q)\right)}\right\| \leq\left((1+\gamma) \lambda_{0}\right)^{n}<\lambda^{n},
$$

which is a contradiction.

2.2. From local maximality to hyperbolicity, $(\mathbf{c}) \Rightarrow(\mathbf{a})$. To prove this part of the Main Theorem we need some additional $C^{1}$-generic statements. Our first theorem asserts that given any two saddles of the same index in a $C^{1}$-generic homoclinic class, one can create intersections between stable and unstable manifolds of these saddles. Theorem 2.6 was mentioned in [2, 6, 16, although it was not precisely formulated. We try to state it explicitly and give a direct proof.

Theorem 2.6. There is a residual subset $\mathcal{R}_{2}$ of $\operatorname{Diff}^{1}(M)$ such that for any $f \in \mathcal{R}_{2}$, if $p$ and $q$ are two saddles of $f$ of the same index contained in a homoclinic class of $f$, then $p \sim q$.

In the proof of Theorem 2.6, we shall apply Lemma 2.7 and Proposition 2.8 below. Hayashi's $C^{1}$-Connecting Lemma (Lemma 2.7) was originally designed as a tool for creating intersections between invariant manifolds of periodic points.

Lemma 2.7 (Hayashi's $C^{1}$-Connecting Lemma 20]). Consider $f \in \operatorname{Diff}^{1}(M)$ with two hyperbolic saddles $p$ and $q$. Assume that there are a sequence $\left(x_{n}\right)$ and natural numbers $k_{n}$ such that

- $x_{n} \rightarrow x \in W_{l o c}^{u}(p, f), x \neq p$, and

- $f^{k_{n}}\left(x_{n}\right) \rightarrow y \in W_{l o c}^{s}(q, f), y \neq q$.

Then there is a diffeomorphism $g$ arbitrarily $C^{1}$-close to $f$ such that $W^{u}\left(p_{g}, g\right)$ and $W^{s}\left(q_{g}, g\right)$ have an intersection arbitrarily close to $x$.

To state the next proposition, we need a definition. For any diffeomorphism $f$ and any natural number $n$, let $\mathrm{SI}_{n}(f)$ be the set of all ordered pairs $(r, s)$ of saddles of $f$ such that

- $r$ and $s$ are contained in the same homoclinic class of $f$,

- $r$ and $s$ have the same index, and

- the periods of $r$ and $s$ are less than $n$.

Proposition 2.8 is a slightly different version of Lemma 2.1 in [2]; however the proof is similar.

Proposition 2.8. There is a residual subset $\mathcal{R}_{3}$ of $\operatorname{Diff}^{1}(M)$ such that any $f \in \mathcal{R}_{3}$ has the following properties:

- $f$ is a Kupka-Smale diffeomorphism.

- For any natural number $n$ there is a neighborhood $\mathcal{U}_{n}$ of $f$ in $\mathcal{R}_{3}$ such that for any $g \in \mathcal{U}_{n}$ the set $\operatorname{SI}_{n}(g)$ is the continuation of the set $\operatorname{SI}_{n}(f)$, which means

$$
S I_{n}(g)=\left\{\left(r_{g}, s_{g}\right) ;\left(r_{f}, s_{f}\right) \in S I_{n}(f)\right\} .
$$

In particular, $\sharp S I_{n}(g)=\sharp S I_{n}(f)<\infty$, where $\sharp$ denotes the cardinal number. 
Proof of Theorem 2.6. Let $\mathcal{G}_{n}$ be the set of all $C^{1}$-diffeomorphisms $f \in \mathcal{R}_{3}$ such that for any $(r, s) \in \mathrm{SI}_{n}(f)$,

$$
W^{u}(r) \bar{\pitchfork} W^{s}(s) \neq \emptyset .
$$

We shall prove that the set $\mathcal{G}_{n}$ is an open and dense subset of $\mathcal{R}_{3}$. By the openness of the transversality condition and the second assertion of Proposition 2.8, the set $\mathcal{G}_{n}$ is already an open subset of $\mathcal{R}_{3}$. To prove the density of $\mathcal{G}_{n}$, consider a diffeomorphism $f \in \mathcal{R}_{3}$ and let $\mathcal{U}_{n}$ be an open neighborhood of of $f$ given by Proposition 2.8. Choose an open neighborhood $\mathcal{V}$ of $f$ in $\operatorname{Diff}^{1}(M)$ such that $\mathcal{V} \cap \mathcal{R}_{3} \subset \mathcal{U}_{n}$. In an inductive process, we find a diffeomorphism $g \in \mathcal{G}_{n} \cap \mathcal{V}$.

Suppose that $(r, s) \in \mathrm{SI}_{n}(f)$. By the transitivity of a homoclinic class, there exist a sequence $\left(x_{n}\right)$ and natural numbers $k_{n}$ satisfying the assumption of the Connecting Lemma. Thus, one can get a diffeomorphism $g_{1} \in \mathcal{V}$ with a transverse intersection between the unstable manifold of $r_{g_{1}}$ and the stable manifold of $s_{g_{1}}$. Since this configuration is robust under $C^{1}$-perturbations, we can, maybe after another small perturbation, assume that $g_{1} \in \mathcal{U}_{n}$. By using the Connecting Lemma again for $g_{1}$ and taking in mind the persistence of the first transverse intersection, one can get a new diffeomorphism $g_{2} \in \mathcal{U}_{n}$ such that the unstable and stable manifolds of another ordered pair in $\mathrm{SI}_{n}\left(g_{2}\right)$ intersect each other transversally, and so on. By the second assertion of Proposition 2.8, this process leads to a diffeomorphism $g \in \mathcal{U}_{n}$ such that for any $(r, s) \in \operatorname{SI}_{n}(g), W^{u}(r) \pitchfork W^{s}(s) \neq \emptyset$, it means that $g \in \mathcal{G}_{n}$. Now, the residual set

$$
\mathcal{R}_{2}=\bigcap_{n \in \mathbb{N}} \mathcal{G}_{n}
$$

has the desired property mentioned in Theorem 2.6.

In 2 the authors proved that the $C^{1}$ generic homoclinic classes are index complete. That is, whenever a generic homoclinic class contains two periodic points of index $\alpha$ and $\beta$ with $\alpha<\beta$, then it contains periodic points of index $\gamma$ with $\gamma \in[\alpha, \beta]$. We rewrite its consequence in our context (Corollary 2 in [2]).

Proposition 2.9. There is a residual subset $\mathcal{R}_{4}$ of $\operatorname{Diff}^{1}(M)$ such that for any $f \in \mathcal{R}_{4}$ and any saddle $p$ of $f$, if the locally maximal homoclinic class $H(p, f)$ contains saddles of different indices, then it contains a saddle of the same index as that of $p$ and with arbitrarily weak hyperbolicity.

Corollary 2.10. There is a residual subset of $\operatorname{Diff}^{1}(M)$ such that for any diffeomorphism $f$ belonging to it, if $f$ has the UHPO property on a locally maximal homoclinic class, then any saddle $q \in H(p, f)$ is homoclinically related to $p$. In particular, any two saddles of $f$ in $H(p, f)$ have the same index.

Proof. Consider a diffeomorphism $f \in \mathcal{R}_{2} \cap \mathcal{R}_{4}$ having the UHPO property on a locally maximal homoclinic class $H(p, f)$. Arguing by contradiction, assume that the homoclinic class contains a saddle $q$ of a different index from that of $p$. Since $f \in \mathcal{R}_{4}, H(p, f)$ also contains a saddle $q$ having the same index as that of $p$ with arbitrarily weak hyperbolicity. As $f \in \mathcal{R}_{2}, q$ is homoclinically related to $p$, which contradicts the UHPO property on $H(p, f)$. Hence, any saddle $q$ of $f$ in $H(p, f)$ has the same index as that of $p$ and is homoclinically related to it. 
We denote by $\mathcal{M}_{f}(\Lambda)$ the set of all $f$-invariant probability measures supported on a compact $f$-invariant set $\Lambda$. As it is known, the set $\mathcal{M}_{f}(\Lambda)$ is a compact set in the weak*-topology. By the Ergodic Decomposition Theorem, the convex combinations of probability ergodic measures is dense in $\mathcal{M}_{f}(\Lambda)$. We recall that a Borel subset of $\Lambda$ is said to have full probability if it has full measure for any $\mu \in \mathcal{M}_{f}(\Lambda)$. Suppose that $E \subset T_{\Lambda} M$ is a continuous direction. For any $x \in M$, let

$$
\lambda(x, E)=\lim _{n \rightarrow \infty} \frac{1}{n} \log \left\|\left.D f^{n}(x)\right|_{E(x)}\right\|
$$

whenever the limit exists. By Oseledec's Theorem, these limits exist on a full probability set and are called Lyapunov exponents of $f$ along $E$.

The next theorem gives us a framework which allows us to unify the notion of UHPO and the non-existence of zero Lyapunov exponents for $C^{1}$-generic locally maximal homoclinic classes.

Theorem 2.11. There is a residual subset of $\operatorname{Diff}^{1}(M)$ such that for any diffeomorphism $f$ that belongs to it, $f$ has the UHPO property on a locally maximal homoclinic class if and only if there is a continuous splitting $E \oplus F$ for the homoclinic class such that the Lyapunov exponents are negative along $E$ and positive along $F$ on a full probability set.

For the proof of Theorem 2.11, we need the $C^{1}$-generic properties mentioned in Proposition 2.12 below. We recall that for a periodic point $p$ of a diffeomorphism $f$ of period $\pi(p)$, the atomic measure $\mu_{p}$ associated to the orbit of $p$ is defined by

$$
\mu_{p}=\frac{1}{\pi(p)} \sum_{n=0}^{\pi(p)-1} \delta_{f^{n}(p)} .
$$

One of the basic properties of ergodic measures is that every $C^{1}$-generic ergodic measure can be approximated arbitrarily well in weak*-topology by periodic measures. This fact is a reformulation of Mañé's Ergodic Closing Lemma, cited below.

Proposition 2.12. There is a residual subset $\mathcal{R}_{5}$ of $\operatorname{Diff}^{1}(M)$ such that any $f \in \mathcal{R}_{5}$ has the following properties:

- $f$ is a Kupka-Smale diffeomorphism,

- for any ergodic measure $\mu$ of $f$, there is a sequence $q_{n}$ of periodic points such

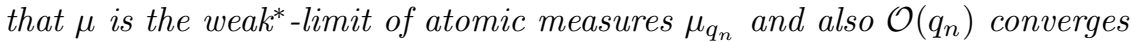
to Supp $\mu$ in Hausdorff metric ([3]).

Proof of Theorem 2.11. Consider a diffeomorphism $f \in \mathcal{R}_{2} \cap \mathcal{R}_{4} \cap \mathcal{R}_{5}$ having the UHPO property on a locally maximal homoclinic class $H(p, f)$ and let $\mu$ be an $f$-invariant ergodic measure on $H(p, f)$. Combining Corollary 2.10 and the second assertion of Proposition 2.12, one can get a sequence $q_{n}$ of periodic points homoclinically related to $p$ such that $\mu_{q_{n}}$ converges to $\mu$ in weak*-topology. For $\mu$-a.e. point $x$, we have

$$
\begin{aligned}
\lim _{n \rightarrow \infty} \frac{1}{n} \log \left\|\left.D f^{n}\right|_{E(x)}\right\| \leq \int \log \left\|\left.D f\right|_{E}\right\| d \mu & =\lim _{n \rightarrow \infty} \int \log \left\|\left.D f\right|_{E}\right\| d \mu_{q_{n}} \\
& =\log \prod_{i=0}^{\pi\left(q_{n}\right)-1}\left\|\left.D f\right|_{E\left(f^{i}\left(q_{n}\right)\right)}\right\| \\
& \leq \log \lambda^{\pi\left(q_{n}\right)} .
\end{aligned}
$$


The first inequality holds by Birkhoff's Ergodic Theorem. By the Ergodic Decomposition Theorem, the same fact holds for any $f$-invariant measure. Similar argumentation can be applied for the direction $F$.

For the converse, suppose that for any $\mu \in \mathcal{M}_{f}(H(p, f))$ and $\mu$-a.e. point $x$, $\lambda_{\mu}(x)=\lim _{n \rightarrow \infty} 1 / n \log \left\|\left.D f^{n}\right|_{E(x)}\right\|<0$. Put $\lambda_{\mu}=\int \lambda_{\mu} d \mu$. By the Sub-additive Ergodic Theorem [28], for any $\mu \in \mathcal{M}_{f}(H(p, f))$,

$$
\lim _{n \rightarrow \infty} \frac{1}{n} \int \log \left\|\left.D f^{n}\right|_{E}\right\| d \mu=\lambda_{\mu}<0 .
$$

Hence, for any $\mu \in \mathcal{M}_{f}(H(p, f))$, there is a natural number $n_{\mu}$ such that

$$
1 / n_{\mu} \int \log \left\|\left.D f^{n_{\mu}}\right|_{E}\right\| d \mu<\lambda_{\mu}+\delta<0
$$

By continuity, the same holds in a neighborhood of $\mu$ in $\mathcal{M}_{f}(H(p, f))$. By the compactness of $\mathcal{M}_{f}(H(p, f))$, there exist a natural number $m>0$ and a constant $0<\lambda<1$ such that

$$
\forall \mu \in \mathcal{M}_{f}(H(p, f)), \exists 1 \leq n_{\mu} \leq m, 1 / n_{\mu} \int \log \left\|\left.D f^{n_{\mu}}\right|_{E}\right\| d \mu<\log \lambda .
$$

In particular, for any saddle $q$ in $H(p, f)$, considering the atomic measure $\mu_{q}$, there is a natural number $1 \leq n_{q} \leq m$ such that $\prod_{i=0}^{\pi(q)-1}\left\|\left.D f^{n_{q}}\right|_{E\left(f^{i}(q)\right)}\right\|<\lambda^{\pi(q)}$. Putting $k=m$ !, we have

$$
\prod_{i=0}^{\pi(q)-1}\left\|\left.D f^{k}\right|_{E\left(f^{i}(q)\right)}\right\|<\lambda^{\pi(q)} .
$$

Using the same argumentation framework for the direction $F$, the UHPO property on $H(p, f)$ can be deduced.

End of the proof of $(\mathrm{c}) \Rightarrow$ (a). Consider a $C^{1}$-generic diffeomorphism $f$ having the UHPO property on a locally maximal homoclinic class $H(p, f)$ and let $E \oplus F$ be the dominated splitting given by Proposition 2.2. It is known that a continuous direction is contracting (expanding) provided that all the Lyapunov exponents along $E$ are negative (positive) on a full probability set ([10, 11, 18). Hence, Theorem 2.11 essentially ends the proof of $(\mathrm{c}) \Rightarrow(\mathrm{a})$. The proof of $(\mathrm{c}) \Rightarrow(\mathrm{a})$ may also be deduced directly from (1) and the following lemma, which is just Lemma I.5 in 22 .

Lemma 2.13. Let $\Lambda$ be a compact invariant set of $f \in \operatorname{Diff}^{1}(M)$ and $E \subset T_{\Lambda} M$ be a continuous invariant direction. If

$$
\int \log \left\|\left.D f\right|_{E}\right\| d \mu<0
$$

for any $f$-invariant ergodic measure $\mu$ on $\Lambda$, then $E$ is contracting.

\section{Some Applications to the ReCEnt Results}

Before proceeding, let us introduce a notion. A diffeomorphism $f$ has a heterodimensional cycle associated to two saddles $p$ and $q$ if $p$ and $q$ have different indices and both intersections $W^{s}(p) \cap W^{u}(q)$ and $W^{s}(p) \cap W^{u}(q)$ are non-empty.

Corollary 3.1. There is a residual subset of $\operatorname{Diff}^{1}(M)$ such that for any diffeomorphism $f$ that belongs to it, any non-trivial locally maximal chain component of $f$ is either hyperbolic or can be approximated by a heterodimensional cycle. 
We borrow some arguments and results in order to prove Corollary 3.1. For this, we need a new definition. Suppose that $\Lambda$ is a compact $f$-invariant set of a diffeomorphism $f$. We say that $\Lambda$ is fundamental $i$-limit if there is a sequence of diffeomorphisms $g_{k}$ that converges to $f$ in the $C^{1}$-topology together with a sequence $p_{k}$, where $p_{k}$ is a periodic point of $g_{k}$ of index $i$, such that $\mathcal{O}\left(p_{n}\right) \rightarrow \Lambda$ in the Hausdorff metric.

Proposition 3.2. There is a residual subset $\mathcal{R}_{6}$ of $\operatorname{Diff}^{1}(M)$ such that any $f \in \mathcal{R}_{6}$ has the following properties:

- any locally maximal chain component is a homoclinic class ([6, 15]),

- any fundamental $i$-limit set of $f$ is a Hausdorff limit of hyperbolic periodic points of $f$ of index $i(29])$,

- if $\Lambda$ is a transitive set of $f$ containing two hyperbolic periodic points of different indices, then a heterodimensional cycle can be created by an arbitrarily small $C^{1}$-perturbation ([17]).

Proof of Corollary 3.1. Consider a diffeomorphism $f \in \mathcal{R}_{2} \cap \mathcal{R}_{4} \cap \mathcal{R}_{6}$ and let $C$ be a non-trivial locally maximal chain component of $f$ in an open subset $U$ of $M$.

First, suppose that all the periodic points in $C$ have the same index. By the second assertion of Proposition 3.2, there is no index changing in $U$ for a diffeomorphism $C^{1}$-close to $f$. More precisely, any periodic point of a diffeomorphism $g C^{1}$-close to $f$ whose orbit is entirely contained in $U$ is hyperbolic. Now, using Mañé's argumentation in 21], the UHPO property of $f$ on $C$ can be deduced. In view of the Main Theorem, the chain component $C$ is hyperbolic.

Now, suppose that $C$ has periodic points of different indices. Then, by the third assertion of Proposition 3.2, it can be approximated by a heterodimensional cycle.

$C^{1}$-generically, any two homoclinic classes are either equal or are completely disjoint (12]). This gives a good characterization of a $C^{1}$-generic diffeomorphism according to the number of its homoclinic classes. A generic diffeomorphism with a finite number of homoclinic classes is called tame, and a generic diffeomorphism with an infinite number of them is called wild. The same as Smale's pieces in the context of uniform hyperbolicity, any homoclinic class of a tame diffeomorphism is locally maximal ([1]). Although the following corollary has been proved in 1 by the other method, our approach also provides a new proof for it. In fact, Corollary 3.3 is already a direct consequence of Corollary 3.1.

Corollary 3.3. $C^{1}$-generically, tame diffeomorphisms are either hyperbolic or can be approximate by a heterodimensional cycle.

In a heterodimensional configuration, if the stable and unstable manifolds of two hyperbolic sets $\Lambda$ and $\Sigma$ with different index intersect each other, then questions may arise: is the heterodimensional robust? which means "do the continuations of $\Lambda$ and $\Sigma$ form a heterodimensional cycle"? Recently, Corollary 3.3 has been extended through a positive answer to the question above. In [7] the authors have proved the following $C^{1}$-dichotomy for tame diffeomorphisms: A $C^{1}$-generic tame diffeomorphism is either hyperbolic or it has a robust heterodimensional cycle.

An invariant compact set $\Lambda$ of a diffeomorphism $f$ is called Lyapunov stable if for any neighborhood $V$ of $\Lambda$ there is a neighborhood $U$ of it such that $f^{n}(U) \subseteq V$, for any natural number $n$. In [7] the authors have shown that if a $C^{1}$-generic Lyapunov 
stable homoclinic class admits a dominated splitting $E \oplus F$ with $\operatorname{dim} E=1$, then the direction $E$ is uniformly contracting. The expansion of the direction $F$ can be obtained from [9]. We can state the following theorem as a consequence.

Theorem 3.4. If $H(p, f)$ is a Lyapunov stable homoclinic class of a diffeomorphism $f \in \mathcal{R}_{1}$ with index $(p)=1$, then the UHPO property on $H(p, f)$ implies its hyperbolicity.

\section{ACKNOWLEDGMENTS}

The author would like to take this opportunity to express his thanks to Shaobo Gan and Meysam Nassiri for their comments and also to ICTP for their hospitality during the author's short visit. This research was supported in part by a grant from IPM (No. 90370038).

\section{REFERENCES}

[1] F. Abdenur, Attractors of generic diffeomorphisms are persistent, Nonlinearity, 16 (2003), 301-311. MR 1950789 (2003k:37040)

[2] F. Abdenur, C. Bonatti, S. Crovisier, L. J. Diaz and L. Wen, Periodic points in homoclinic classes, Ergod. Th. \& Dynam. Sys., 27 (2007), 1-22. MR2297084 (2008k:37048)

[3] F. Abdenur, C. Bonatti and S. Crovisier, Non-uniform hyperbolicity for $C^{1}$-generic diffeomorphisms, Israel J. of Math., 183 (2011), 1-60. MR2811152

[4] J. Alves, V. Araújo and B. Saussol, On the uniform hyperbolicity of some nonuniformly hyperbolic systems, Proc. Amer. Math. Soc., 131 (2003), 1303-1309. MR.1948124 (2003k:37046)

[5] V. Araújo, Non-zero Lyapunov exponents, no sign changes and Axiom A, arXiv:math/ 0403273v2 [math.DS], latest version 20 Feb 2011 (v6).

[6] C. Bonatti and S. Crovisier, Récurrence et généricité, Invent. Math., 158 (2004), 33-104. MR2090361 (2007b:37036)

[7] C. Bonatti and L.J. Diaz, Robust heterodimensional cycles and $C^{1}$-generic dynamics, J. de l'Institute de Math. de Jussieu, 7 (3) (2008), 469-523. MR2427422 (2009f:37020)

[8] C. Bonatti, L. Diaz and M. Viana, Dynamics beyond uniform hyperbolicity. A global geometric and probabilistic perspective, Encyclopedia of Mathematical Sciences, 102. Mathematical Physics III. Springer-Verlag, Berlin (2005). MR2105774 (2005g:37001)

[9] C. Bonatti, S. Gan and D. Yang, On the hyperbolicity of homoclinic classes, Disc. \& Cont. Dynam. Sys., 25, 4 (2009), 1143-1162. MR2552132(2011b:37046)

[10] Y. Cao, Non-zero Lyapunov exponents and uniform hyperbolicity, Nonlinearity, 16 (2003), 1473-1479 MR.1986306 (2005g:37061)

[11] Y. Cao, S. Luzzatto and S. Rios, Uniform hyperbolicity for random maps with positive Lyapunov exponents, Proc. Amer. Math. Soc., 136 (2008), 3591-3600. MR2415043(2010h:37118)

[12] C. Carballo, C. Morales and M. J. Pacífico, Homoclinic classes for generic $C^{\perp}$ vector fields, Ergod. Th. \& Dynam. Sys., 23 (2003), 403-415. MR.1972228 (2004e:37031)

[13] A. Castro, New criteria for hyperbolicity based on periodic sets, Bull. Braz. Math. Soc., New Series, 42 (3) (2011), 455-483. MR2833813 (2012g:37062)

[14] A. Castro, K. Oliveira and V. Pinheiro, Shadowing by nonuniformly hyperbolic periodic points and uniform hyperbolicity, Nonlinearity (Bristol), 20 (2007), 75-85. MR.2285105 (2007k:37023)

[15] S. Crovisier, Periodic orbits and chain-transitive sets of $C^{1}$-diffeomorphisms, Publ. Math. Inst. Hautes Études Sci., 104 (2006), 87-141. MR2264835(2008f:37047)

[16] S. Crovisier, M. Sambarino and D. Yang, Partial hyperbolicity and homoclinic tangency, preprint.

[17] S. Gan and L. Wen, Heteroclinic cycles and homoclinic closures for generic diffeomorphisms, J. Dyn. and Diff. Equ., 15, 2-3 (2003), 451-471. MR2046726 (2005b:37029)

[18] B. Hasselblatt, Y. Pesin and J. Schmeling, Pointwise hyperbolicity implies uniform hyperbolicity, Oberwolfach preprint (OWP) 06 (2009).

[19] S. Hayashi, Diffeomorphisms in $\mathcal{F}^{1}(M)$ satisfy Axiom A, Ergod. Th. \& Dynam. Sys., 12 (1992), 233-253. MR1176621 (94d:58081) 
[20] S. Hayashi, Connecting invariant manifolds and the solution of the $C^{1}$-stability and $\Omega$ stability conjectures for flows, Ann. of Math., 145 (1997), 81-137. MR1432037 (98b:58096)

[21] R. Mañé, An ergodic closing lemma, Ann. of Math., 116 (1982), 503-540. MR678479 (84f:58070)

[22] R. Mañé, A proof of the $C^{1}$ stability conjecture, Inst. Hautes Et́udes Sci. Publ. Math., 66 (1988), 495-524. MR932138 (89e:58090)

[23] R. Potrie and M. Sambarino, Codimension one generic homoclinic classes with interior, Bull. Braz. Math. Soc., New Series, 41 (1) (2010), 125-138. MR2609214 (2011c:37062)

[24] R. Potrie, Generic bi-Lyapunov stable homoclinic classes, Nonlinearity, 23 (2010), 1631-1649. MR2652474(2011h:37030)

[25] E. Pujals and M. Sambarino, On the dynamics of dominated splitting, Ann. of Math. (2), 169 (2009), no. 3, 675-739. MR2480616 (2010b:37087)

[26] E. Pujals and F. R. Hertz, Critical points for surface diffeomorphisms, J. Modern Dyn., 1 (4) (2007), 1-34. MR2342701 (2008k:37092)

[27] K. Sakai, Diffeomorphisms with $C^{2}$ stable shadowing, Dynam. Sys., 17 (2002), 235-241. MR 1927810 (2003f:37046)

[28] P. Walters, An introduction to ergodic theory, Springer-Verlag, New York-Berlin (1982). MR648108 (84e:28017)

[29] L. Wen, Generic diffeomorphisms away from homoclinic tangencies and heterodimensional cycles, Bull. Braz. Math. Soc., New Series, 35 (2004), 419-452. MR2106314 (2005i:37035)

[30] D. Yang and S. Gan, Expansive homoclinic classes, Nonlinearity, 22 (2009), 729-733. MR2486353(2010b:37055)

Faculty of Mathematical Sciences, Shahid Beheshti University, Tehran, Iran

Current address: School of Mathematics and Computer Sciences, Damghan University, P. O. Box 36715-364, Damghan, Iran - and - School of Mathematics, Institute for Research in Fundamental Sciences (IPM), P. O. Box 19395-5746, Tehran, Iran

E-mail address: fakhari@du.ac.ir, abs.fakhari@gmail.com 